\title{
Luminescence of Acceptors in Mg-Doped GaN
}

Bo Monemar, Sergey Khromov, Galia Pozina, Plamen Paskov, Peder Bergman, Carl

Hemmingsson, Lars Hultman, Hiroshi Amano, Vitaliy Avrutin, Xing Li and Hadis Morkoc

\section{Linköping University Post Print}

\section{Tweet}

N.B.: When citing this work, cite the original article.

Original Publication:

Bo Monemar, Sergey Khromov, Galia Pozina, Plamen Paskov, Peder Bergman, Carl Hemmingsson, Lars Hultman, Hiroshi Amano, Vitaliy Avrutin, Xing Li and Hadis Morkoc, Luminescence of Acceptors in Mg-Doped GaN, 2013, Japanese Journal of Applied Physics, (52), 8 .

http://dx.doi.org/10.7567/JJAP.52.08JJ03

Copyright: Japan Society of Applied Physics http://www.jsap.or.jp/

Postprint available at: Linköping University Electronic Press http://urn.kb.se/resolve?urn=urn:nbn:se:liu:diva-98151 


\section{Luminescence of acceptors in Mg-doped GaN}

Bo Monemar ${ }^{1}$, Sergey Khromov ${ }^{1}$, Galia Pozina ${ }^{1}$, Plamen Paskov ${ }^{1}$, Peder Bergman ${ }^{1}$, Carl Hemmingsson ${ }^{1}$, Lars Hultman ${ }^{1}$, Hiroshi Amano ${ }^{2}$, Vitaliy Avrutin ${ }^{3}$, Xing Li $^{3}$, Hadis Morkoç ${ }^{3}$ ${ }^{1}$ Linköping University, S-581 83 Linköping, Sweden ${ }^{2}$ Nagoya University, Chikusa-ku, Nagoya, 464-8603, JAPAN

${ }^{3}$ Virginia Commonwealth University, Richmond, Virginia 23284, USA

Recent photoluminescence (PL) data for Mg-doped GaN at $2 \mathrm{~K}$ are discussed, with reference to published theoretical calculations of the electronic level structure. It is concluded that the typical PL peaks at $3.466 \mathrm{eV}$ (acceptor bound exciton ABE1) and the broader $3.27 \mathrm{eV}$ donor-acceptor pair (DAP) PL are the expected standard PL signatures of the substitutional Mg acceptor. Additional broader peaks at $3.455 \mathrm{eV}$ (ABE2) and $3.1 \mathrm{eV}$ are suggested to be related tot he same acceptors perturbed by nearby basal plane stacking faults. The low temperature metastability of PL spectra is assigned to a nonradiative metastable deep level. 
Optical experiments on semiconductors give the most detailed information on the energy level structure of dopants via their characteristic spectra, and also on the mechanisms of recombination of carriers or excitons via these energy levels. Recent photoluminescence (PL) studies show that Mg-doping in GaN produces at least two acceptor species A1 and A2 with different optical signatures ${ }^{1}$. This has been found from careful work on low dislocation density homo-epitaxial layers with a quite low dislocation density $\left(<10^{7} \mathrm{~cm}^{-2}\right)$. The PL spectra for an acceptor typically consists of a narrow neutral acceptor bound exciton (ABE) line and a broad donor-acceptor pair (DAP) spectrum at lower energies. In this case the A1 PL at low $\mathrm{T}$ consists of the $3.466 \mathrm{eV} \mathrm{ABE} 1$ and the $3.27 \mathrm{eV}$ DAP, while the A2 signatures are ABE2 at $3.454 \mathrm{eV}$ and the $3.1 \mathrm{eV}^{\mathrm{DAP}}{ }^{1}$. The possible identity of these two acceptors has been discussed in previous work ${ }^{1-3}$, but not clarified. In hetero-epitaxial samples grown on sapphire, with a much higher structural defect density, more than two acceptor states were recently reported for GaN: $\mathrm{Mg}^{4}$. The situation with more than one acceptor for each dopant is unusual in semiconductors, and its explanation calls for more detailed experimental investigations. Recent structural studies of $c$-plane and $m$-plane GaN:Mg layers grown by MOCVD on low defect bulk GaN substrates reveal a high density of nano-size basal plane stacking faults (BSFs) in such material, induced by the Mg-doping,6. The interaction of the substitutional Mg acceptors with these SFs may explain the presence of the two shallow acceptor states, and their optical properties.

The following identification of the two Mg-related acceptors is suggested here: A1 is the isolated substitutional $\mathrm{Mg}$ acceptor, while A2 is the same acceptor interacting with a nearby basal plane SF. (Note that this assignment is modified from the one in Ref. 1). Arguments in support of this are: The A1 PL spectra dominate at low Mg doping $\left(<10^{18} \mathrm{~cm}^{-3}\right)$, where the material is n-type and no SFs are seen. In fact no other shallow acceptor species is observed in optical spectra for such material. (This argues against the suggestion that the $3.27 \mathrm{eV}$ PL is 
related to a neutral defect state, that should only be present for a Fermi level position close to the valence band ${ }^{3}$ ). The presence of two closely spaced peaks for ABE1 is consistent with expectations for such an ABE state ${ }^{7}$. The ABE2 PL is only strong at high Mg doping $\left(>10^{18}\right.$ $\mathrm{cm}^{-3}$ ), when nano-size BSFs also exist ${ }^{5.6}$. A spatial correlation between $\mathrm{Mg}$ atoms and these BSFs has been shown in TEM studies ${ }^{5}$. The ABE2 PL shows a strong broadening (Fig. 1), consistent with inhomogeneous broadening with a slight variation of the distance to the SF and the size of the SF (local strain broadening). The BSFs also show their own characteristic luminescence spectra in cathodoluminescence $(C L)^{5}$, and also in PL (Fig. 1). The peaks present in the region $3.38-3.42 \mathrm{eV}$ in Fig. 1, also previously reported in Ref. 8, are similar to BSF-related CL spectra recently observed in nanostructured GaN samples ${ }^{9}$.

The identity of the $3.27 \mathrm{eV}$ spectrum as a DAP recombination has been well documented in the literature. There is a strong shift with excitation intensity as well as with decay time, typical for a DAP with a distribution of donor-acceptor distances ${ }^{10}$, and a transition from a DAP to conduction band-acceptor spectrum with elevated temperature has been clearly demonstrated $^{11,12}$. The broadening of this spectrum towards lower energy at high Mg doping can be explained as a stronger localization of the A2 type Mg acceptors that are associated with BSFs.

The deeper so called blue PL spectrum peaking around $2.9 \mathrm{eV}$ is present strongly in highly Mg-doped MOVPE grown GaN, but seems to be typically absent in similar samples grown with $\mathrm{MBE}^{13}$. An identification as a DAP band involving deep acceptors and shallow donors is supported by ODMR data ${ }^{14}$, but the defect corresponding to the $2.9 \mathrm{eV}$ PL could also be a neutral complex. The peak seems to be H-related, and the corresponding defect does not noticeably affect the hole concentration ${ }^{15}$. These results argue against the recent suggestion that the $2.9 \mathrm{eV}$ PL band is the signature of the regular substitutional $\mathrm{Mg}$ acceptor in $\mathrm{GaN}^{3}$. In such a case the $2.9 \mathrm{eV}$ PL would dominate at all Mg concentrations, and for all growth techniques. 
The metastability data in PL was discussed in some detail in Ref. 1, where these effects were tentatively explained by the presence of a Mg-H complex acceptor as the identity of acceptor A1. It may be explained in a different way, however, as related to a deeper metastable non-radiative defect which has no PL signature, but affects the quasi-Fermi level position upon UV excitation, in particular before annealing. In Fig. 2 PL spectra are shown in the DAP region for different times of prolonged UV excitation of an Mg-doped sample with [Mg] about $1 \times 10^{19} \mathrm{~cm}^{-3}$ before anneal. There is a clear downshift of the PL peak with excitation time, meaning that the quasi-Fermi level is upshifted in the bandgap to lower the hole occupancy of the $\mathrm{Mg}$ acceptor. A deeper level may then possess the metastable properties ${ }^{1}$, governing the quasi-Fermi level during this experiment. This has the important consequence that it allows the identification of $\mathrm{A} 1$ as the substitutional $\mathrm{Mg}$ acceptor, as argued above. Indeed the A1 PL signatures are present at both low and high Mg-doping, and exhibit optical properties typical for moderately shallow acceptors in wide bandgap materials, if the metastability can be assigned to a separate deep level defect.

Acknowledgements The Linköping partners are grateful for financing of spectroscopic equipment from the K. A. Wallenberg Foundation, and for project support from the Li-Li NFM Center at Linköping University. 


\section{References}

1) B. Monemar, P. P. Paskov, G. Pozina, C. Hemmingsson, J. P. Bergman, T. Kawashima, H. Amano, I. Akasaki, T. Paskova, S. Figge, D. Hommel, and A. Usui, Phys. Rev. Lett. 102 (2009) 235501.

2) S. Lany and A. Zunger, Appl. Phys. Lett. 96 (2010) 142114.

3) J.L. Lyons, A. Janotti, and C. G. Van de Walle, Phys. Rev. Lett. 108 (2012) 156403.

4) G. Callsen, M. R. Wagner, T. Kure, J. S. Reparaz, M. Bügler, J. Brunnmeier, C. Nenstiel, A. Hoffmann, M. Hoffmann, J. Tweedie, Z. Bryan, S. Aygun, R. Kirste, R. Collazo, and Z. Sitar, Phys. Rev. B 86 (2012) 075207.

5) S. Khromov, C. G. Hemmingsson, H. Amano, B. Monemar, L. Hultman, and G. Pozina, Phys. Rev. B 84 (2011) 075324.

6) S. Khromov, B. Monemar, V. Avrutin, X. Li, H. Morkoç, L. Hultman, and G. Pozina, Appl. Phys. Lett. 100 (2012) 172108.

7) B. Gil, P. Bigenwald, P. P. Paskov, and B Monemar, Phys. Rev. B 81 (2010) 085211.

8) R. Stepniewski, A. Wysmolek, M. Potemski, J. Lusakowski, K. Korona, K. Pakula, J.M. Baranowski, G. Martinez, P. Wyder, I. Grzegory, and S. Porowski, Phys. Stat. Sol. B 210 (1998) 373.

9) J. Lähnemann,, O. Brandt, U. Jahn, C.Pfüller, C. Roder,P. Dogan, F. Grosse, A. Belabbes, F. Bechstedt, A. Trampert, and L. Geelhaar, Phys. Rev. B 86 (2012) 081302.

10) R. Dingle and M. Ilegems, Solid State Commun. 5 (1971) 175.

11) O. Lagerstedt and B. Monemar, J. Appl. Phys. 45 (1974) 2266.

12) M. A. Reshchikov, D. Huang, F. Yun, L. He, and H. Morkoc, Appl. Phys. Lett. 79 (2001) 3779. 
13) E.R,Glaser, M. Murthy, J. A. Freitas Jr., D. F. Storm, L. Zhou, and D. J. Smith, Physica B 401-402 (2007) 327.

14) E. R. Glaser, W. E. Carlos, G. C. B. Braga, J. A. Freitas Jr., W. J. Moore, G. V. Shanabrook, R. L. Henry, A. E. Wickenden, D. D. Koleske, H. Obloh, P. Kozodoy, S. P. DenBaars, and U. K. Mishra, Phys. Rev. B 65 (2002) 085312.

15) Y. Kamiura, M. Kaneshiro, J. Tamura, T. Ihiyama, Y.Yamashita, T. Mitani, and T. Mukai, Jpn. J. Appl. Phys. 44 (2005), L 926-L 928. 


\section{Figure captions.}

Fig.1. (Color online) Near bandgap PL spectra of Mg-doped m-plane GaN. Peaks around 3.4 $\mathrm{eV}$ may be SF related.

Fig.2. Spectral redshift of the $3.27 \mathrm{eV}$ DAP PL emission vs long time continuous UV excitation at $2 \mathrm{~K}$ before anneal for a sample with $[\mathrm{Mg}]$ about $1 \times 10^{19} \mathrm{~cm}^{-3}$. 


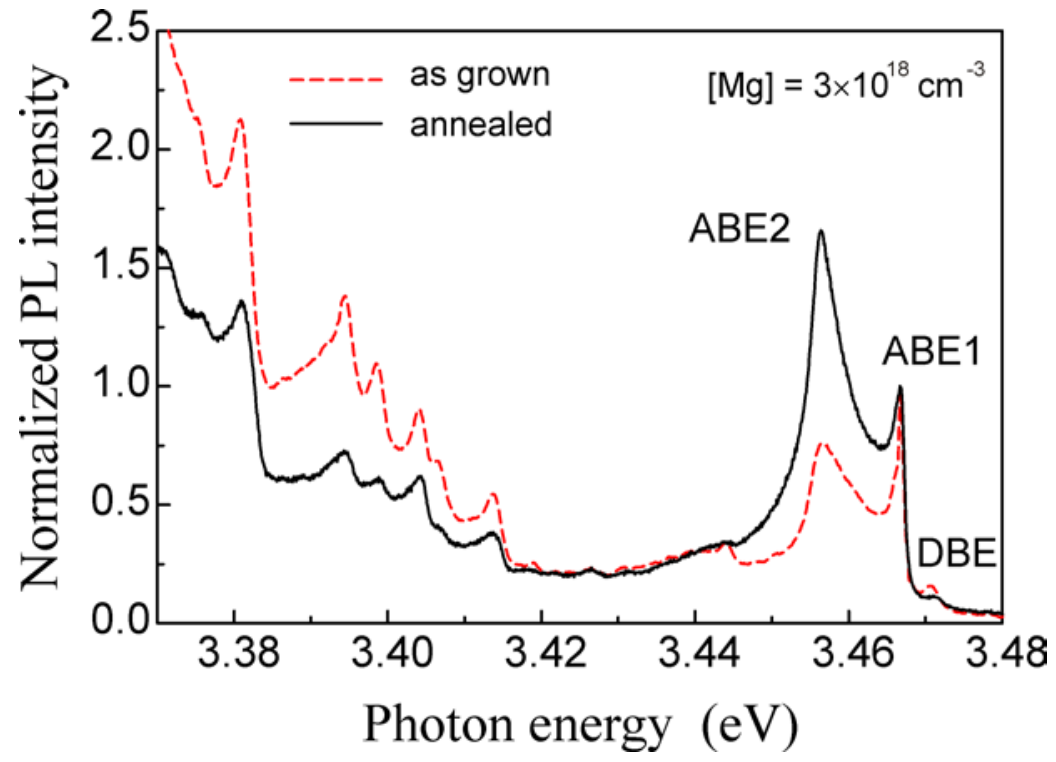

Fig 1. 


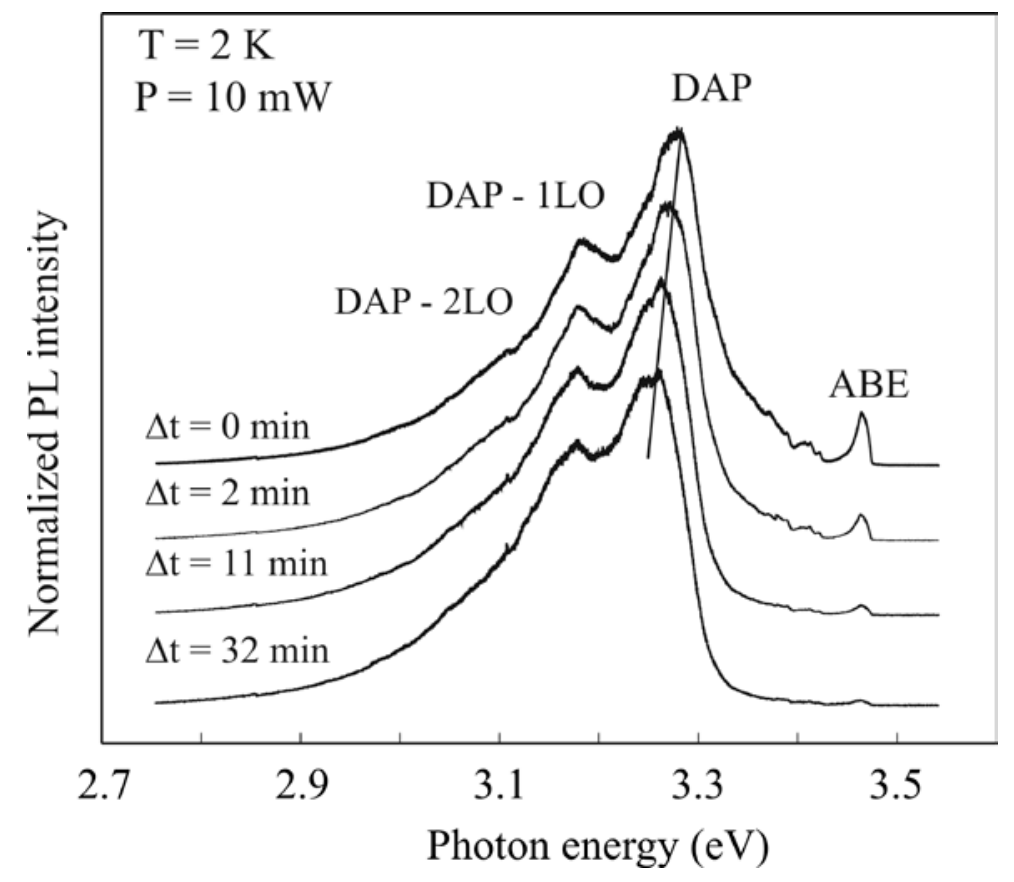

Fig.2 